\title{
Effect of Coadministration of Etonogestrel Implant and Antiretroviral Therapies Containing Efavirenz or Lopinavir/Ritonavir on the Metabolism of Women Living with HIV/AIDS
}

Tatiana R Rocha Prandini', Maria Valeria Bahamondes ${ }^{2,3}$, Milena B Brito ${ }^{4}$, Eliana Amaral ${ }^{3}$, Luis Bahamondes ${ }^{2,3}$, Geraldo Duarte ${ }^{1}$, Silvana M Quintana', Rui A Ferriani ${ }^{1,2}$ and Carolina Sales Vieira ${ }^{1,3 *}$

${ }^{1}$ Department of Gynecology and Obstetrics, Medical School of Ribeirao Preto, University of São Paulo, Brazil

${ }^{2}$ National Institute of Hormones and Women's Health, Brazil

${ }^{3}$ Department of Obstetrics and Gynecology, Faculty of Medical Sciences, University of Campinas, Brazil

${ }^{4}$ Department of Gynecology and Obstetrics, Federal University of Bahia, Brazil

\begin{abstract}
Background: Concomitant use of antiretroviral therapy (ART) and hormonal contraceptives should be safe for women while maintaining medication efficacy without causing significant immunological or metabolic changes. However, the metabolic effect of the concomitant use of etonogestrel (ENG)-releasing implant and commonly used
\end{abstract} ARTs is not known.

Objective: To evaluate the effect of coadministration of ENG-releasing implant and ARTs containing lopinavir/ ritonavir or efavirenz on the metabolic parameters of women living with human immunodeficiency virus (HIV) infection/ acquired immune deficiency syndrome (AIDS).

Methods: This is an open, prospective, non-randomized exploratory study. Forty-five women who sought to receive the ENG contraceptive implant were selected, including 15 non-ART users, 15 zidovudine/lamivudine (AZT/3TC)+lopinavir/ritonavir users and $15 \mathrm{AZT} / 3 \mathrm{TC}+$ efavirenz users for at least 3 months. Clinical and metabolic parameters were assessed before, during and 24 weeks after implant insertion.

Results: At 24 weeks after ENG implant insertion, the group without ART showed a $4.7 \%$ increase in albumin level $(p=0.03)$, a $6.2 \%$ decrease in total cholesterol level $(p=0.03)$ and a $5.6 \%$ increase in alkaline phosphatase level $(p<0.01)$, whereas the lopinavir/ritonavir group showed a $12.5 \%$ increase in aspartate aminotransferase $(p=0.03)$. The efavirenz group showed a $12.9 \%$ decrease in low-density lipoprotein $(p=0.03)$. The other results showed no significant changes during the 24 week study. The observed changes remained within the normal values for the parameters.

Conclusion: Co-administration of ENG implant with ARTs containing lopinavir/ritonavir or efavirenz is not associated with clinically relevant metabolic changes in women living with HIV infection/AIDS, after a 6 month followup period.

Keywords: Etonogestrel implant; HIV; Contraception; Lipid profile; Antiretroviral therapy; Efavirenz

\section{Introduction}

Unplanned pregnancy is a health problem affecting all countries, particularly developing countries [1]. In vulnerable populations, the rate of unplanned pregnancies is even higher than that of general population. In Brazil, the rate of unplanned pregnancies among women living with HIV infection/AIDS exceeds 70\% [2], whereas this rate is $55.4 \%$ among the general population [3]. Adequate contraception prevents unintended pregnancies, unsafe abortions, and maternal and newborn deaths, and helps to prevent vertical transmission of HIV infection [4].

Family planning of women living with HIV infection/AIDS has a direct effect on their health and is a key strategy of the World Health Organization (WHO) to reduce vertical transmission [5]. In Brazil, the vertical transmission rate decreased to $33.3 \%$ in the last 10 years [6]. However, considering the high rates of unplanned pregnancy among these women, investment in family planning could contribute to further decrease vertical transmission rates. A $50 \%$ decrease in the number of births among women living with HIV infection/AIDS would be associated with a decrease from $27 \%$ to $5 \%$ in vertical transmission rate, resulting in an approximately $90 \%$ decrease in the number of children with HIV infection [7].
In Brazil, since 2013, the number of people using antiretroviral therapy (ART) increased to $30 \%$, and most of this population is of reproductive age [8]. Thus, the safety of hormonal contraceptive coadministration with the ARTs most often used by women living with HIV infection/AIDS must be known when prescribing hormonal contraceptives to these women. A recent study that evaluated the pharmacokinetic interaction between the two ART regimens most commonly used in Brazil showed that co-administration of etonogestrel (ENG) implant, the most effective contraceptive available, with ART containing lopinavir/ritonavir resulted in a 52\% increase in ENG bioavailability, whereas co-administration with ART containing

*Corresponding author: Carolina Sales Vieira, MD, PhD, Department of Obstetrics and Gynecology, School of Medicine of Ribeirao Preto/USP, Av. Bandeirantes 3900, Campus Universitário, Ribeirao Preto, SP 14049-900, Brazil; Tel: +55 16 3602-2804; Fax: +55 16 3633-0946; E-mail: carol.sales@uol.com.br

Received February 16, 2017; Accepted March 06, 2017; Published March 13, 2017

Citation: Prandini TRR, Bahamondes MV, Brito MB, Amaral E, Bahamondes L, et al (2017) Effect of Coadministration of Etonogestrel Implant and Antiretroviral Therapies Containing Efavirenz or Lopinavir/Ritonavir on the Metabolism of Women Living with HIVIAIDS. J AIDS Clin Res 8: 674. doi: 10.4172/2155-6113.1000674

Copyright: (C) 2017 Prandini TRR, et al. This is an open-access article distributed under the terms of the Creative Commons Attribution License, which permits unrestricted use, distribution, and reproduction in any medium, provided the original author and source are credited. 
Citation: Prandini TRR, Bahamondes MV, Brito MB, Amaral E, Bahamondes L, et al. (2017) Effect of Coadministration of Etonogestrel Implant and Antiretroviral Therapies Containing Efavirenz or Lopinavir/Ritonavir on the Metabolism of Women Living with HIVIAIDS. J AIDS Clin Res 8: 674. doi: 10.4172/2155-6113.1000674

efavirenz (EFV) caused a $63 \%$ decrease in ENG bioavailability. The ovulation rate in the group of EFV users was 3\%, albeit without pregnancies [9]. Subsequently, an observational study showed that the incidence of pregnancies was three times higher among users of ART containing EFV while using any hormonal contraceptive, whereas the use of an ENG implant remained the most effective method among the hormonal treatments tested (pills and injectable), despite the decreased efficacy [10].

No studies that evaluated the metabolic safety of co-administration of ENG implants with any ART have been published. Thus, the present study aimed to evaluate the metabolic safety (e.g. lipid profile, glucose levels and inflammatory marker and hepatic and kidney markers) of co-administration of ENG implant with ARTs containing EFV or lopinavir/ritonavir.

\section{Methods \\ Participants}

This is a secondary and exploratory analysis of a pharmacokinetic study [9], with a prospective, non-randomized, controlled design. The sample included 15 HIV-positive women who received zidovudine/lamivudine (AZT/3TC)+lopinavir/ritonavir (LPV/r) for at least 3 months (LPV/r group), $15 \mathrm{HIV}$-positive women treated with AZT/3TC+EFV for at least 3 months (EFV group) and 15 HIV-positive women who did not use ART (non-ART group) because they failed to meet the clinical criteria for indication of ART at the time of the recruitment. The criteria recommended for ART initiation in Brazil at the time of patient recruitment was a CD4 count of $<350$ cells $/ \mathrm{mm}^{3}$ [11].

The study was conducted at the Clinical Research Unit of the Hospital das Clínicas of the Medical School of Ribeirão Preto and the Department of Obstetrics and Gynecology of the School of Medical Sciences of the Universidade de Campinas, Brazil. Participants were recruited from family planning outpatient clinics and HIV/AIDS treatment centers from September 2010 to April 2012. The team of researchers conducted an active search for medical records and files on ART prescription filling in the pharmacies of all centers attending HIV-positive patients in both cities. After the pre-screening, patients who met the inclusion criteria were invited to participate in the study. The project was approved by the research ethics committees of both institutions, and all volunteers signed the informed consent form.

Women who met the following criteria were included in the study: willing to sign the informed consent form, aged between 18 and 45 years, sought to use the ENG implant, had regular menstrual cycles (21-35 days), body mass index between 18 and $30 \mathrm{~kg} / \mathrm{m}^{2}$, at least 60 days from fullterm pregnancy, HIV-1-positive according to the criteria of the Ministry of Health [12] and undergoing stable ART for at least 3 months (only in the groups of ART users). Conversely, the following exclusion criteria were used: medical contraindications (category 3 or 4) for ENG implant insertion according to the WHO eligibility criteria [13], delivery in the last 42 days, use of hormonal contraception in the last 60 days, use of depot medroxyprogesterone acetate in the last 6 months, acute or opportunistic diseases requiring treatment in the last 30 days, alcohol or drug addiction, uncontrolled clinical disease, use of drugs metabolized by CYP3A4, chronic diarrhea or malabsorption syndromes (ART only groups) and non-adherence to ART (ART only groups).

\section{Study protocol}

The participants returned for ENG implant insertion in the first 5 days of the cycle (or at any day of the cycle after a negative pregnancy test) and after fasting for 8 hours. The implant was inserted in the inner side of the non-dominant arm by using the standardized technique. Before inserting the implant, the volunteers were subjected to venipuncture to collect whole blood, requiring $20 \mathrm{~mL}$ for the metabolic study. This sampling was repeated 24 weeks after inserting the ENG implant.

The following anthropometric variables were also measured: systolic (SBP) and diastolic (DBP) blood pressure, measured in a sitting position with a mercury sphygmomanometer on the left arm after resting for $\pm 20 \mathrm{~min}$; abdominal circumference, defined as the smallest measurement between the lateral iliac crest and the inferior margin of the last rib; and height and weight (to calculate the body mass index $[\mathrm{BMI}])$. Data on the viral load assay and CD4/CD8 cells are reported in the previously published pharmacokinetic study [9].

The metabolic safety was evaluated by measuring the effects of the ENG-implant on the lipid profile, blood glucose levels, inflammatory marker (C-reactive protein) and on the hepatic and kidney markers.

The lipid profile variables (total cholesterol, high-density lipoprotein [HDL] and triglycerides) were assessed with the enzymatic method by using the Wiener liquid line Colestat AA enzymatic kit (Wiener Laboratory, Rosario, Argentina). Low-density lipoprotein (LDL) cholesterol was calculated by using the Friedewald formula [14].

C-reactive protein (CRP) level was measured by using chemiluminescence with the DPC Immulite 1000 and 2000 immunoassay analyzers (Diagnostic Products Corporation, Los Angeles, CA, USA). The hepatic markers assessed were gammaglutamyl transferase (gamma-GT), alkaline phosphatase (ALP), albumin, total and direct bilirubin, aspartate transaminase (AST), and alanine transaminase (ALT) levels. The modified Szasz method was used for gamma-GT determination with the Wiener $\gamma$-L-AA kinetic test kit (Wiener Laboratory). ALP level was measured by using the optimized kinetic method with the Wiener ALP kit 405 liquid AA (Wiener Laboratory). Albumin level was measured by using the colorimetric method with the AA Albumin kit (Wiener Laboratory). Total and direct bilirubin measurements were performed by using a photometric test, and indirect bilirubin level was calculated by subtracting the direct bilirubin level from the total. Levels of the liver enzymes AST and ALT were measured by using the optimized ultraviolet (UV) method IFCC (International Federation of Clinical Chemistry) and the Wiener AST (SGOT) AA liquid kit (Wiener Laboratory) and Wiener ALT (SGPT) liquid AA kit (Wiener Laboratory). The chemistry analyzer used to measure hepatic markers was Konelab 60i (Thermo Fisher Scientific, Finland).

Renal markers, urea, and creatinine were measured with the kinetic UV method by using Wiener AA urea liquid kit (Wiener Laboratory) and Wiener creatinine AA liquid kit (Wiener Laboratory). The chemical analyzer used was Konelab 60i (Thermo Fisher Scientific).

Glucose oxidase level was determined by Konelab $60 \mathrm{i}$ chemical analyzer (Thermo Fisher Scientific) and the Wiener AA enzyme glucose liquid kit (Wiener Laboratory). The intra- and inter-assay coefficients of variation were lower than $6 \%$.

\section{Statistical analysis}

Sample size calculation was performed for pharmacokinetic evaluation [9]. The same sample was used for metabolic assessment because this is a secondary data analysis, thus for this outcome it is considered an exploratory analysis. 
Citation: Prandini TRR, Bahamondes MV, Brito MB, Amaral E, Bahamondes L, et al. (2017) Effect of Coadministration of Etonogestrel Implant and Antiretroviral Therapies Containing Efavirenz or Lopinavir/Ritonavir on the Metabolism of Women Living with HIV/AIDS. J AIDS Clin Res 8: 674. doi: 10.4172/2155-6113.1000674

Page 3 of 5

The linear mixed-effects (random and fixed effects) regression model was used to compare quantitative variables, except viral load (VL). Comparisons were adjusted for age, which differed between the groups. The nonparametric Wilcoxon (intragroup) or Kruskal-Wallis (intergroup) test was used to analyze for VL. The nonparametric tests were performed with $\mathrm{R}$ software (R Foundation for Statistical Computing, Vienna, Austria), and the other statistical tests were performed with the SAS 9.0 software (SAS Institute Inc., Cary, NC, USA). The variables were analyzed based on intention to treat (ITT) and the last-observation-carried-forward method was used for the missing variables. The significance level adopted was 0.05 .

\section{Results}

One woman from each group was excluded from the study. Of the 15 women who started the study in the EFV group, one woman was excluded because of changes in her ART at week 4 . In the LPV/r group, one woman had pulmonary tuberculosis and had to be excluded from the group because she changed her ART at week 12. In the non-ART group, one woman had to start ART at week 20 of the study and was subsequently excluded (Figure 1).

The mean age of the EFV group was higher than that of the other groups. The other baseline variables showed no significant differences among the groups (Table 1). Of the immunological variables, only VL was higher in non-ART group than in other groups $(\mathrm{p}<0.01)$ in the period before ENG implant insertion. BMI and blood pressure were not different among groups in baseline evaluation.

At 24 weeks after ENG implant insertion, albumin level increased by $4.7 \%(\mathrm{p}=0.03)$, total cholesterol level decreased by $6.2 \%(\mathrm{p}=0.03)$, ALP level increased by $5.6 \%(\mathrm{p}<0.01)$, diastolic blood pressure decreased by $0.7 \%(\mathrm{p}=0.04)$ and abdominal circumference $(\mathrm{AC})$ increased by $1.2 \%$ $(\mathrm{p}=0.02)$ in non-ART group. In the same period, EFV group showed a $12.9 \%$ decrease in LDL level $(\mathrm{p}=0.03)$ and $\mathrm{LPV} / \mathrm{r}$ group showed a $12.5 \%$ increase in AST level ( $\mathrm{p}=0.03)$, whereas the other variables did not present significant changes during the 24 weeks of ENG implant use in each group (Table 2). The described changes in study variables remained within the normal limits.

\section{Discussion}

Co-administration of ENG implant with the ARTs tested (regimens containing LPV/r and EFV) caused no deleterious changes in the metabolic profiles of women living with HIV. The evaluation of multiple variables in this study is a needed step to increase the evidence of safety outcomes of the co-administration of ENG implant with the ARTs tested (regimens containing LPV/r and EFV). This information is also necessary to guide healthcare providers and women during contraceptive counseling and decision-making process.

In EFV group, LDL level decreased by $12.9 \%$ at 6 months of ENG implant use, whereas no significant changes in any lipid profile variable occurred in the same period of observation in LPV/r group. This is an interesting finding because increased total cholesterol and triglyceride levels are potential metabolic effects of both EFV and LPV/r therapies [15-17]. Combined hormonal contraceptives reportedly increased total cholesterol, triglyceride and HDL levels, and decreased LDL levels in women who were non-users of these medications and HIV negative [18]. Conversely, the use of progestogen contraceptives alone is not associated with metabolic abnormalities or significant increase

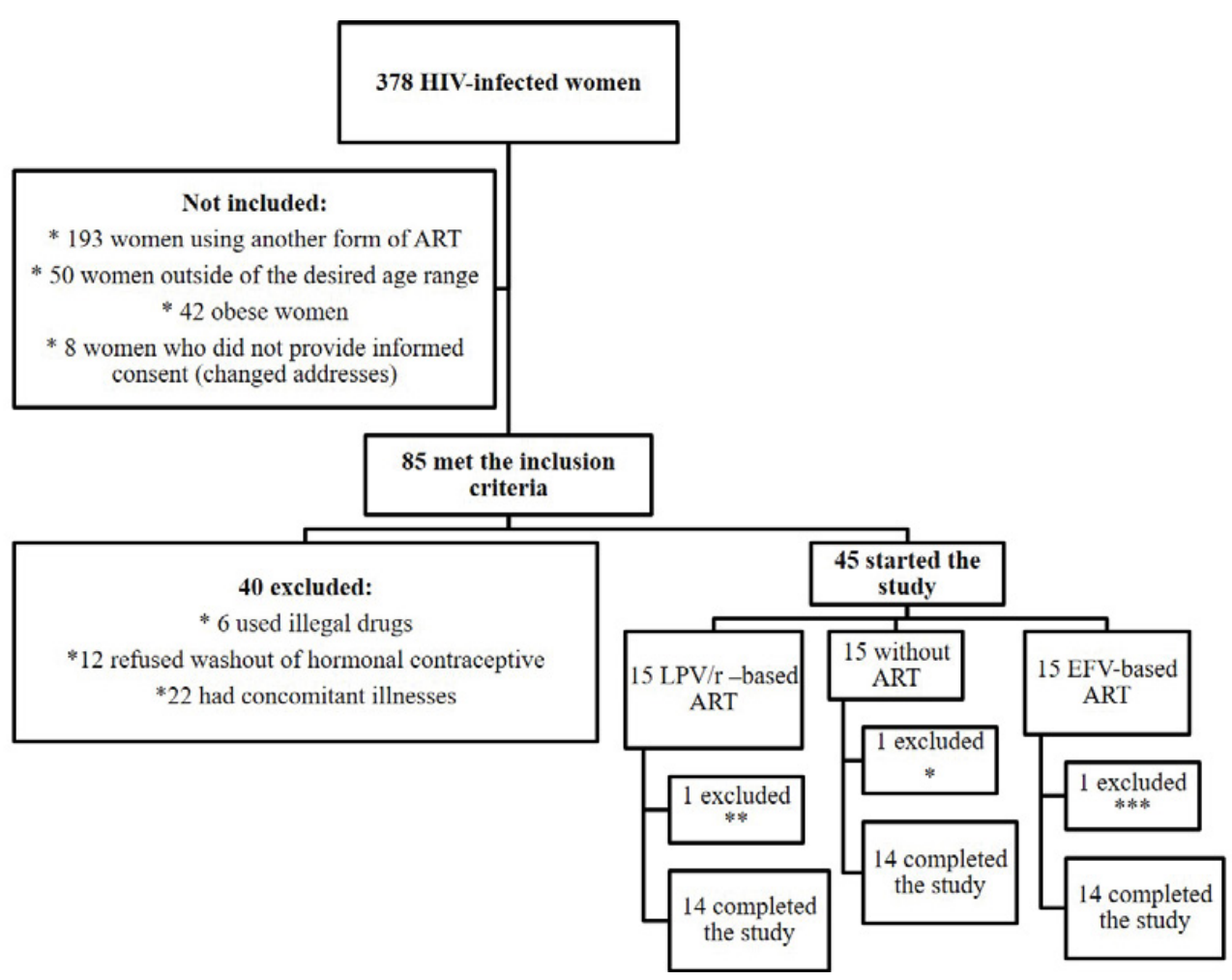

ART: Antiretroviral Therapy; LPV/r-based ART: Zidovudine/lamivudine (AZT/3TC)+lopinavir/ritonavir; EFV-based ART: AZT/3TC+efavirenz, * Started use of ART after week 20. ${ }^{* *}$ Had pulmonary tuberculosis, which resulted in a change in the ART regimen after week $12 .{ }^{* * *}$ Changed ART regimen after week 4

Figure 1: Flowchart of the study. 
Citation: Prandini TRR, Bahamondes MV, Brito MB, Amaral E, Bahamondes L, et al. (2017) Effect of Coadministration of Etonogestrel Implant and Antiretroviral Therapies Containing Efavirenz or Lopinavir/Ritonavir on the Metabolism of Women Living with HIVIAIDS. J AIDS Clin Res 8: 674. doi: 10.4172/2155-6113.1000674

Page 4 of 5

\begin{tabular}{|c|c|c|c|}
\hline & Without ART & LPV/r Group & EFV Group \\
\hline Age (years) & $28.8 \pm 6.2^{\star}$ & $27.1 \pm 3.7^{*}$ & $34.5 \pm 6.2^{*}$ \\
\hline Education (years) & $10.7 \pm 3.7^{\star}$ & $7.7 \pm 3.4^{*}$ & $8.2 \pm 3.6^{*}$ \\
\hline Tobacco smokers (\%) & $33.3 \%(5 / 15)$ & $26.7(4 / 15)$ & $33.3 \%(5 / 15)$ \\
\hline Parity (>3 childbirths) & $27 \%(4 / 15)$ & $20 \%(3 / 15)$ & $27 \%(4 / 15)$ \\
\hline Family income (Brazilian reals) & $1412.9 \pm 1065.1$ & $1400 \pm 1324.1$ & $1418.6 \pm 971.6$ \\
\hline
\end{tabular}

Quantitative variables are expressed as mean ( \pm standard deviation)

${ }^{*} p<0.01$, other comparisons showed a $p$ value of $>0.05$

Table 1: Clinical baseline characteristics of the HIV-positive women who were receiving and those who were not receiving antiretroviral therapy (zidovudine/ lamivudine+lopinavir/ritinavir or zidovudine/lamivudine+efavirenz).

\begin{tabular}{|c|c|c|c|c|c|c|}
\hline & \multicolumn{2}{|c|}{ Without ART } & \multicolumn{2}{|c|}{ LPV/r Group } & \multicolumn{2}{|c|}{ EFV Group } \\
\hline & 0 & 24 weeks & 0 & 24 weeks & 0 & 24 weeks \\
\hline $\mathrm{SBP}(\mathrm{mm} \mathrm{Hg})$ & $114.5(104-124.5)$ & $103(100-121.5)$ & $112(108-120)$ & $110.5(100-130)$ & $120(110-126)$ & $109(104-112)$ \\
\hline DBP (mm Hg) & $70.5(67-76.5)$ & $69.5(60-73.5)^{*}$ & $71.5(65-80)$ & $70(69-79)$ & $80(66-82)$ & 72 (69-81) \\
\hline$A C(\mathrm{~cm})$ & $85.5(75-89.5)$ & $86.5(84-91.5)^{*}$ & $78.5(73-87)$ & $78.5(75-89)$ & $82(72.5-95)$ & $86(75-98)$ \\
\hline BMI $\left(\mathbf{k g} / \mathrm{m}^{2}\right)$ & $23.7(20.6-26.3)$ & $23(21.2-27.5)$ & $25(22.3-28)$ & $26.3(23-17.8)$ & $24.5(20.6-26.3)$ & $25.2(19.1-27.4)$ \\
\hline CD8 (cells/mm³) & $953.5(798-1153)$ & $880.5(731-1060)$ & $756.5(642-1461)$ & 835 (619-1236) & $835.5(654-1027)$ & $809.5(631-1075)$ \\
\hline CD4 (cells/mm³) & $546.5(474-754)$ & 575 (468-710) & 382.5 (224-983) & 466 (314-711) & $745.5(488-821)$ & 727 (507-862) \\
\hline VL (copies/mL) & $2897(743-8886)$ & $4374(484-11764)$ & $<50(<50-1094)$ & $<50(<50-700)$ & $<50(<50-50)$ & $<50(<50-50)$ \\
\hline Glycemia (mg/dL) & $80(74.5-86.5)$ & $84.5(75-90.5)$ & $76.5(73-84)$ & 78.5 (74-89) & 87 (80-95) & $87(73-94)$ \\
\hline Albumin (mg/dL) & $4.2(3.8-4.5)$ & $4.4(4.3-4.5)^{\star}$ & $4.3(4.2-4.4)$ & $4.4(4.3-4.5)$ & $4.25(4.1-4.4)$ & $4.1(4.0-4.3)$ \\
\hline Total Bb (mg/dL) & $0.39(0.24-0.72)$ & $0.4(0.29-0.59)$ & $0.36(0.27-0.46)$ & $0.4(0.27-0.66)$ & $0.26(0.22-0.34)$ & $0.24(0.22-0.28)$ \\
\hline Direct Bb (mg/dL) & $0.13(0.07-0.24)$ & $0.13(0.07-0.22)$ & $0.11(0.08-0.15)$ & $0.14(0.11-0.19)$ & $0.09(0.07-0.13)$ & $0.09(0.07-0.11)$ \\
\hline Indirect Bb (mg/dL) & $0.29(0.14-0.48)$ & $0.27(0.15-0.48)$ & $0.23(0.16-0.33)$ & $0.41(0.17-0.49)$ & $0.15(0.14-0.21)^{c}$ & $0.17(0.1-0.19)$ \\
\hline Urea (mg/dL) & $24(19-27)$ & $21(18-31)$ & $25(22-34)$ & $26(21-29)$ & $23.5(20-29)$ & $19.5(16-27)$ \\
\hline Creatinine (mg/dL) & $0.71(0.68-0.9)$ & $0.75(0.66-0.83)$ & $0.79(0.72-0.84)$ & $0.8(0.73-0.87)$ & $0.78(0.7-0.8)$ & $0.76(0.72-0.83)$ \\
\hline $\mathrm{TC}(\mathrm{mg} / \mathrm{dL})$ & 177 (165-195) & $166(130-215)^{*}$ & $163(143-189)$ & $148.5(138-174)$ & 179 (163-199) & $161(150-174)$ \\
\hline TG (mg/dL) & $140(107-201)$ & $138(82-187)$ & $111(67-150.5)$ & $74(46-122)$ & $89(70-115)$ & $106.5(70-133)$ \\
\hline HDL (mg/dL) & $42(39-50)$ & $42(34-47)$ & $45(36-53)$ & $38(31-49)$ & $48.5(44-57)$ & $45(41-52)$ \\
\hline LDL (mg/dL) & $104(93.4-119.8)$ & $99.6(78.6-132)$ & $92.6(75.5-118.4)$ & $95.6(70.2-107.2)$ & $110.3(104.8-121.4)$ & $96(88-111.6)^{*}$ \\
\hline AST (U/L) & $19.9(16.8-26.5)$ & $16.9(15.5-28.6)$ & 16 (11.4-18.2) & $18(14.5-24.4)^{*}$ & $17.3(16.3-20.1)$ & $17.3(15.4-23.3)$ \\
\hline ALT (U/L) & $14.3(8.4-18.3)$ & $12.3(8.6-15.8)$ & $13.4(7.3-17.5)$ & $12.8(8.7-16.2)$ & $8.35(7-13.4)$ & $9.6(7.8-13.2)$ \\
\hline Gamma GT (U/L) & $22(19-41)$ & $29(19-44)$ & $20(15-30)$ & $24(18-40)$ & $27.5(22-37)$ & $33(24-42)$ \\
\hline ALP (U/L) & 179 (154-209) & $189(161-267)^{*}$ & $150(111-178)$ & $129(121-169)$ & $151.5(121-195)$ & $169.5(140-181)$ \\
\hline CRP (mg/dL) & $0.24(0.18-0.56)$ & $0.29(0.17-0.36)$ & $0.15(0.12-0.45)$ & $0.23(0.07-0.37)$ & $0.29(0.16-1.01)$ & $0.5(0.13-0.92)$ \\
\hline
\end{tabular}

The variables are expressed as median (first and third quartile)

${ }^{*} p<0.05$, compared with the baseline (time 0 )

SBP: Systolic Blood Pressure; DBP: Diastolic Blood Pressure; AC: Abdominal Circumference; BMI: Body Mass Index; VL: Viral Load; Bb: Total Bilirubin; TC: Total Cholesterol; TG: Triglycerides; HDL: High-Density Lipoprotein; LDL: Low-Density Lipoprotein; AST: Aspartate Transaminase; ALT: Aspartate Transaminase; Gamma GT: Gamma Glutamyl Transferase; ALP: Alkaline Phosphatase; CRP: C-Reactive Protein

Table 2: Intra-group comparison of the effects of etonogestrel-releasing implants on the clinical and metabolic variables in HIV-positive women who were receiving and those who were not receiving antiretroviral therapy (zidovudine/lamivudine+lopinavir/ritinavir or zidovudine/lamivudine+efavirenz).

in cardiovascular risk [19]. The use of ENG-releasing implants was associated with a decrease in total cholesterol and HDL levels in the 6 months of observation of women living without HIV infection [20]

Co-administration of ENG implants with the ARTs tested was associated with a $12.5 \%$ increase in AST level in the group of LPV/r users. Most likely, this fact is attributed not only to the presence of the ENG implant but also to other factors because no increase occurred in implant users without ART. The LPV/r association may increase liver enzyme levels [16]. ENG-releasing implants have been associated with a non-significant slight decrease in AST and ALT among HIV-negative women [21]. Increased total bilirubin level secondary to increased levels of indirect bilirubin has already been shown in ENG implant users $[21,22]$. However, all the changes in liver markers reported in this study and in previous studies remained within their normal limits.

The non-ART group, which expressed the effect of the ENG implant on metabolic variables in HIV-positive women, showed a $4.7 \%$ increase in albumin level, 5.6\% increase in creatinine level, $6.2 \%$ decrease in total cholesterol level, 5.6\% decrease in AF, $1.4 \%$ decrease in diastolic blood pressure, and $1.2 \%$ increase in AC. It is noteworthy that all changes in this group were lower than $10 \%$ of the baseline and remained within the normal ranges. In the HIV-negative women who received an ENGreleasing implant, studies have shown a decrease in total cholesterol and HDL levels in the 6 months of evaluation [20] and increased total bilirubin level secondary to increased indirect bilirubin levels [22].

A noticeable limitation of this study is the secondary analysis of a pharmacokinetic study. Therefore, the sample size may be small for metabolic assessment of the ENG implant association with the ARTs tested. The clinical relevance of the changes at 6 months may be questionable because the changes found were mostly $<10 \%$ of the preinsertion values. Another limitation is the follow-up period. The fact that the coadministration of ENG implant with the ARTs tested failed to show any significant negative change at 6 months precludes ensuring the same results in longer follow-up studies. Further studies should be conducted to assess the metabolic safety of the association of these drugs for longer periods. 
Citation: Prandini TRR, Bahamondes MV, Brito MB, Amaral E, Bahamondes L, et al. (2017) Effect of Coadministration of Etonogestrel Implant and Antiretroviral Therapies Containing Efavirenz or Lopinavir/Ritonavir on the Metabolism of Women Living with HIVIAIDS. J AIDS Clin Res 8: 674. doi: 10.4172/2155-6113.1000674

Page 5 of 5

However, it should be noted that the use of progestogen-only contraceptives and non-hormonal contraceptives is recommended for women with cardiovascular risk factors, thereby avoiding the effects of increased cardiovascular risk generated by combined hormonal contraceptives [23]. The use of progestogen-only contraceptives has a lower risk of thromboembolic events [19,24], causes no changes in blood pressure [18] and may be indicated in most chronic diseases [23].

The strengths of the study included the groups, which were standardized for a pharmacokinetic study [9], with a significantly homogeneous population. No significant change in BMI was observed, no drug that could alter the metabolism of ENG or ARTs was used, and no comorbidities were observed in any of the patients during the 24 week study.

\section{Conclusion}

In conclusion, despite some changes in metabolic variables upon co-administration of ENG implant and ARTs containing EFV or LPV/r, all these changes remained within the normal ranges, thus showing metabolic safety at 6 months of observation. Further studies with higher numbers of subjects and longer follow-up period are necessary to confirm sustained long-term safety.

\section{Conflicts of Interest and Source of Funding}

Carolina Sales Vieira, Rui A Ferriani, Milena B Brito, and Luis Bahamondes give occasional lectures for MSD and Bayer. The present study was co-funded by the São Paulo Research Foundation (Fundação de Amparo à Pesquisa do Estado de São Paulo [FAPESP]) and the Science and Technology Department of the Brazilian Health Ministry (Grant No. 2009/53147-3).

\section{Acknowledgement}

The study was co-funded by the São Paulo Research Foundation (Fundação de Amparo à Pesquisa do Estado de São Paulo [FAPESP]) and the Science and Technology Department of the Brazilian Health Ministry (Grant No. 2009/53147-3). The authors thank the staff of the Clinical Research Unit of the Clinical Hospital of the Schools of Medicine of Ribeirao Preto and Campinas for the care provided to the study volunteers. They also acknowledge the Municipal Health Secretary of Ribeirão Preto of the STD/AIDS Department for supporting the study at the centers for HIV treatment.

\section{References}

1. Singh S, Darroch JE (2012) Adding it up: Costs and benefits of contraceptive services-estimates for 2012. Guttmacher Institute and United Nations Population Fund, New York (NY) 4.

2. Brandão K de S, Lima BG, Travassos AG, de Brito Fde O, de Souza EX, et al (2015) Dual contraception adherence among HIV-infected women. Rev Bras Ginecol Obstet 37: 486-491.

3. Viellas EF, Domingues RMSM, Dias MAB, Gama SGN, Filha MMT, et al. (2014) Prenatal care in Brazil. Cad Saúde Pública 30: S1-S15.

4. World Health Organization (2015) Family planning/contraception fact sheet 351.

5. World Health Organization (2015) Strategic vision 2010-2015: Preventing mother-to-child transmission of HIV to reach the UNGASS and Millennium Development Goals (PMTCT).

6. Ministério da Saúde (2015) Boletim Epidemiológico HIV-AIDS 2015.

7. World Health Organization (2015) Global monitoring framework and strategy for the global plan towards the elimination of new HIV infections among children by 2015 and keeping their mothers alive (EMTCT). World Health Organization, Geneva.

8. Ministétrio da Saúde [Ministry of Health] Notícias AISA (2015) ONU reconhece avanços do país no controle da AIDS

9. Vieira CS, Bahamondes MV, de Souza RM, Brito MB, Rocha Prandini TR, et al.
(2014) Effect of antiretroviral therapy including lopinavir/ritonavir or efavirenz on etonogestrel-releasing implant pharmacokinetics in HIV-positive women. J Acquir Immune Defic Syndr 66: 378-385.

10. Patel RC, Onono M, Gandhi M, Blat C, Hagey J, et al. (2015) Pregnancy rates in HIV-positive women using contraceptives and efavirenz-based or nevirapinebased antiretroviral therapy in Kenya: A retrospective cohort study. Lancet HIV 2: e474-e482.

11. Ministétrio da Saúde (2013) Clinical protocol and therapeutic guidelines for adults living with HIVIAIDS

12. Secretaria de Vigilância em Saúde [Health Surveillance Secretariat] (2009) PORTARIA SVS/MS No 151, DE 14 DE OUTUBRO DE 2009.

13. World Health Organization (2017) Medical Eligibility Criteria for Contraceptive Use. (4th edn) World Health Organization, Geneva.

14. Friedewald WT, Levy RI, Fredrickson DS (1972) Estimation of the concentration of low-density lipoprotein cholesterol in plasma, without use of the preparative ultracentrifuge. Clin Chem 18: 499-502.

15. Haubrich RH, Riddler SA, DiRienzo AG, Komarow L, Powderly WG, et al. (2009) Metabolic outcomes in a randomized trial of nucleoside, non-nucleoside and protease inhibitor-sparing regimens for initial HIV treatment. AIDS 23: 1109-1118.

16. Murphy RL, Brun S, Hicks C, Joseph J, Roy G, et al. (2001) ABT-378/ritonavir plus stavudine and lamivudine for the treatment of antiretroviral-naive adults with HIV-1 infection: 48 week results. AIDS 15: F1-F9.

17. Walmsley S, Bernstein B, King M, Arribas J, Beall G, et al. (2002) Lopinavirritonavir versus nelfinavir for the initial treatment of HIV infection. New Engl Med 346: 2039-2046.

18. Morin-Papunen L, Martikainen $\mathrm{H}$, McCarthy $\mathrm{MI}$, Franks $\mathrm{S}$, Sovio $\mathrm{U}$, et al. (2008) Comparison of metabolic and inflammatory outcomes in women who used contraceptives and the levonorgestrel-releasing intrauterine devices in a general population. Am J Obst Gynecol 199: 529.e1-529.e10.

19. Lidegaard $\varnothing$, Løkkegaard $E$, Jensen A, Skovlund CW, Keiding N (2012) Thrombotic stroke and myocardial infarction with hormonal contraception. N Engl J Med 366: 2257-2266.

20. Dilbaz B, Ozdegirmenci O, Caliskan E, Dilbaz S, Haberal A (2010) Effect of etonogestrel implant on serum lipids, liver function tests and hemoglobin levels. Contraception 81: 510-514

21. Egberg N, van Beek A, Gunnervik C, Hulkko S, Hirvonen E, et al. (1998) Effects on the hemostatic system and liver function in relation to Implanon and Norplant. A prospective randomized clinical trial. Contraception 58: 93-98.

22. Biswas A, Biswas S, Viegas OA (2004) Effect of etonogestrel subderma contraceptive implant (Implanon) on liver function tests: A randomized comparative study with Norplant implants. Contraception 70: 379-382.

23. World Health Organization (2017) Medical eligibility criteria for contraceptive use (5th edn) World Health Organization, Geneva.

24. Tepper NK, Whiteman MK, Marchbanks PA, James AH, Curtis KM (2016) Progestin-only contraception and thromboembolism: A systematic review. Contraception 94: 678-700. 